\title{
WEIGHTED NORM ESTIMATES FOR OBLIQUE DERIVATIVE PROBLEMS
}

\author{
G. M. TROIANIELLO
}

(Communicated by Barbara Lee Keyfitz)

\begin{abstract}
Estimates of the Caccioppoli-Schauder type are proven for a class of regular oblique derivative problems in Hölder function spaces with weights. Previous restrictions on the range of admissible weights are showed to be unnecessary.
\end{abstract}

\section{Notation}

Let $\omega$ be a bounded open subset of a finite-dimensional Euclidean space. We adopt traditional notation for (real) function spaces such as $C^{k}(\bar{\omega}), C^{k, \delta}(\bar{\omega})$, $C_{c}^{k}(\omega), L^{p}(\omega)$, which need not be illustrated. We do however specify that $W^{k, p}(\omega)$ denotes the Sobolev space of order $k$ and exponent $p$ on $\omega$. Next, as in [1], we set

$$
\begin{aligned}
H_{0}(\omega) & =C^{0}(\bar{\omega}), \\
H_{a}(\omega) & =C^{k, a-k}(\bar{\omega}), \quad|\cdot|_{0 ; \omega}=\text { norm in } C^{0}(\bar{\omega}), \\
& \text { for } k \text { (a nonnegative integer) }<a \leq k+1,
\end{aligned}
$$

and denote by $H_{a ; \omega}^{(-b)}, b \leq a$, the family of functions $u: \omega \rightarrow \mathbb{R}$ with finite norms

$$
|u|_{a ; \omega}^{(-b)}=\sup _{\sigma>0} \sigma^{a-b}|u|_{a ; \omega_{\sigma}},
$$

$\omega_{\sigma}$ being the set of points of $\omega$ whose distance from $\partial \omega$ is $>\sigma$. Of course,

$$
H_{a}^{(-a)}(\omega)=H_{a}(\omega), \quad H_{a}^{(-b)}(\omega) \subset H_{a}^{\left(-b^{\prime}\right)}(\omega) \quad \text { if } b^{\prime}<b .
$$

Let

$$
\begin{gathered}
B^{+}=\left\{x \in \mathbb{R}^{N}:|x|<1, x_{N}>0\right\}, \\
S^{0}=\left\{x \in \mathbb{R}^{N}:|x|<1, x_{N}=0\right\}, \quad S^{+}=\partial B^{+} / S^{0}, \\
H_{a}^{(-b)}=H_{a}^{(-b)}\left(B^{+}\right), \quad|\cdot|_{a}^{(-b)}=|\cdot|_{a ; B^{+}}^{(-b)} .
\end{gathered}
$$

Received by the editors August 8, 1993 and, in revised form, January 27, 1994.

1991 Mathematics Subject Classification. Primary 35D10, 35J25; Secondary 35B45, 35B65, $46 \mathrm{E} 35$. 
On the space of functions $u \in H_{a}^{(-b)}$ with supports in the half-sphere $\frac{1}{2} \bar{B}^{+}$of radius $1 / 2$ a norm equivalent to $|u|_{a}^{(-b)}$ is given by $\sup _{\sigma>0} \sigma^{a-b}|u|_{a ; B_{\text {[o] }}^{+}}$, where $B_{[\sigma]}^{+}=\left\{x \in B^{+}:|x|<1, x_{N}>\sigma\right\}$; from [1] and [6] we know that

$$
|u|_{a^{\prime}}^{(-b)} \leq C|u|_{a}^{(-b)}
$$

provided that $0 \leq a^{\prime} \leq a, b \leq a^{\prime}$, and $b<a^{\prime}$ if $b$ is an integer, whereas

$$
|u|_{\mathrm{BMO}} \leq C|u|_{a}^{(0)}
$$

$\left(|\cdot|_{\mathrm{BMO}}=\right.$ norm on the space of functions of BMO, that is, Bounded Mean Oscillation, over $\left.B^{+}\right)$. The above implies $u \in L^{2}\left(B^{+}\right)$for any $b \geq 0$, whereas, if $-1<b<0, u \in L^{p}\left(B^{+}\right)$whatever $p \in[1,-1 / b[$.

\section{STATEMENTS OF THE MAIN RESUlts}

Let $L=-a^{i j} \partial^{2} / \partial x_{i} \partial x_{j}$ (summation convention), where $\left[a^{i j}\right]$ is a positive definite symmetric $N \times N$ real matrix.

For functions $u$ in $H_{a}^{(-b)}$ with supports in $\frac{1}{2} \bar{B}^{+}$it was proven in [1] that the estimate

$$
|u|_{a}^{(-b)} \leq C\left(|u(\cdot, 0)|_{b ; S^{0}}+|L u|_{a-2}^{(2-b)}\right)
$$

holds true provided that $0<b \leq a, a>2$, and neither $a$ nor $b$ is an integer. In [6] it was later proven that (2) remains valid for $b \in \mathbb{N}$ as well. More exactly: let $u_{0}$ be given in $H_{b} ; s^{0}$ and $f^{1}, \ldots, f^{N}$ in $H_{a-1}^{(1-b)}$ for some $b>0$ and some noninteger $a \geq b, a>1$. If a function $u$ in $W^{1, p}\left(B^{+}\right), p=p(b)$, vanishes outside of $\frac{1}{2} \bar{B}^{+}$and satisfies

$$
u(\cdot, 0)=u_{0}, \quad \int_{B^{+}} a^{i j} u_{x_{i}} v_{x_{j}} d x=\int_{B^{+}} f^{i} v_{x_{i}} d x \quad \forall v \in C_{c}^{1}\left(B^{+}\right),
$$

then $u$ is in $H_{a}^{(-b)}$ with

$$
|u|_{a}^{(-b)} \leq C\left(\left|u_{0}\right|_{b ; s^{0}}+\sum\left|f^{i}\right|_{a-1}^{(1-b)}\right) .
$$

(In [6] the case when $a$ is in $\mathbb{N}$ is studied as well, but here we will not dwell upon it.)

Let us pass to the Neumann problem. In [2] it was proven that, whenever $1<b \leq a, a>2$, and neither $a$ nor $b$ is an integer, the estimate

$$
|u|_{a}^{(-b)} \leq C\left(\left|u_{x_{N}}(\cdot, 0)\right|_{b-1 ; S^{0}}+|L u|_{a-2}^{(2-b)}\right)
$$

holds true for any function $u$ in $H_{a}^{(-b)}$ with support in $\frac{1}{2} \bar{B}^{+}$. Notice that for $b$ not an integer (4) is equivalent to the bound

$$
|u|_{b} \leq C\left(\left|u_{x_{N}}(\cdot, 0)\right|_{b-1 ; S^{0}}+|L u|_{a-2}^{(2-b)}\right) .
$$

On one hand, indeed, the implication $(4) \Rightarrow(5)$ is trivially consequent on (1), which yields

$$
|u|_{b}^{(-b)} \leq C|u|_{a}^{(-b)}
$$

since $b$ is not an integer. On the other hand, $u(\cdot, 0) \in H_{b ; S^{0}}$ is the trace on $S^{0}$ of a function $U \in H_{a}^{(-b)}$ which vanishes near $S^{+}$, with

$$
|U|_{a}^{(-b)} \leq C|u(\cdot, 0)|_{b ; S^{0}} \leq C|u|_{b} .
$$


This follows from [1, Lemma 2.3]-a result which we shall return to in a short while. For the moment suffice it to say that, as a consequence, (5) yields (4) once $(2)$ has been applied with $u$ replaced by $u-U$, and consequently $u(\cdot, 0)$ by 0 .

If $b$ is an integer $\geq 2$, counterexamples show that (6) is false in general, and so is (5); yet in the present paper we prove that (4) still holds. More specifically, we have the two following results.

Theorem 1. Let $\left[A^{i j}\right]$ be an $N \times N$ matrix with symmetric part $\left[a^{i j}\right]$. For some $b \geq 1$ and some noninteger $a \geq b$ let $u \in W^{1,2}\left(B^{+}\right)$have support in $\frac{1}{2} \bar{B}^{+}$and satisfy

$$
\int_{B^{+}} A^{i j} u_{x_{i}} v_{x_{j}} d x=\int_{B^{+}} f^{i} v_{x_{i}} d x \quad \forall v \in C^{1}\left(\bar{B}^{+}\right)
$$

where the $f^{i}, s, i=1, \ldots, N$, are functions in $H_{a-1}^{(1-b)}$. Then $u \in H_{a}^{(-b)}$, and the following estimate holds:

$$
|u|_{a}^{(-b)} \leq C \sum\left|f^{i}\right|_{a-1}^{(1-b)}
$$

Theorem 2. Let $g \in H_{b-1 ; S^{0}}, f \in H_{a-2}^{(2-b)}$ for some integer $b \geq 2$ and some noninteger $a>b$. Let $u \in W^{2,2}\left(B^{+}\right)$have support in $\frac{1}{2} \bar{B}^{+}$and satisfy

$$
u_{x_{N}}(\cdot, 0)=g \quad \text { on } S^{0}, \quad L u=f \quad \text { in } B^{+} .
$$

Then $u$ is in $H_{a}^{(-b)}$ with

$$
|u|_{a}^{(-b)} \leq C\left(|g|_{b-1 ; S^{0}}+|f|_{a-2}^{(2-b)}\right) .
$$

Our approach makes crucial use of the extension technique dealt with in the next section, which enables us to follow, in the proof of Theorem 1, the main idea lying behind the above proof that $(5) \Rightarrow(4)$.

We sketchily mention that a perturbation argument, based on considerations similar to those of [1, Proposition 4.3], would allow us to extend our results to a class of uniformly elliptic operators $L$ with variable coefficients, as well as to replace the condition upon $\left.u_{x_{N}}\right|_{S^{0}}$ with one upon $B u=\left.\left(b^{i} u_{x_{i}}+b^{0} u\right)\right|_{S^{0}}$ with $b^{0}, b^{1}, \ldots, b^{N}$ sufficiently regular, $b^{N}>0$. The passage to more general oblique derivative problems

$$
L u=f \quad \text { in } \Omega, \quad B u=g \quad \text { on } \partial \Omega
$$

when $\Omega$ is a bounded domain would then proceed along the lines of [1, Theorem 5.1] and [2, Theorem 2] (which rely upon [1, Lemma 2.6]), provided that $\partial \Omega$ is "sufficiently" regular-more than just Lipschitz continuous, anyway. The Lipschitz case is dealt with in [3] and [4], where $H_{2}^{(-1-\gamma)}(\Omega)$ estimates are proven for $\gamma$ in a suitable range $] 0, \gamma_{0}\left[, 0<\gamma_{0}<1\right.$. The estimate of [4], in particular, is deduced from the main result of that paper, which asserts: If a function is harmonic in $\Omega$ and has an oblique derivative of BMO over $\partial \Omega$, then all its first derivatives are also of BMO over $\partial \Omega$. Such a result does not yield the aforesaid estimate for $\gamma=0$, as is clear from the proof of [4, Theorem 3.1]. Our Theorem 1, instead, does yield an $H_{2}^{(-1)}$ estimate for a function $u$ satisfying (7), hence also $A^{i N} u_{x_{i}}=g$ on $S^{0}$ with $g \in C^{0}\left(S^{0}\right)$, when $f^{N}$ is 
a function from $H_{1+\delta}^{(0)}$ which equals $g$ on $S^{0}$ and is obtained through the extension procedure of [1, Lemma 2.3]. (As for $\gamma=1$ : We know that $H_{2}^{(-2)}$ estimates do not hold, no matter how smooth $\partial \Omega$ is, whereas $H_{2+\delta}^{(-2)}$ estimates hold for every $\delta>0$ as a consequence of our Theorem 2.)

\section{AN EXTENSION LEMMA}

Let $\phi: \mathbb{R}^{N-1} \rightarrow \mathbb{R}$ be a smooth function with compact support, and let $v=$ $v\left(x^{\prime}, x_{N}\right)$ (with the notation $\left.\left(x^{\prime}, x_{N}\right)=x=\left(x_{1}, \ldots, x_{N-1}, x_{N}\right)\right)$ be at least continuous for $x_{N} \geq 0$, with support in $\frac{1}{2} \bar{B}^{+}$. For $t, x_{N} \geq 0$ set

$$
W\left(x^{\prime}, t, x_{N}\right)=\int v\left(x^{\prime}-t y^{\prime}, x_{N}\right) \phi\left(y^{\prime}\right) d y^{\prime} \text {. }
$$

(Here and in the sequel $\int$ stands for $\int_{\mathbb{R}^{n-1}}$.) By [1, Lemma 2.3] the function

$$
V\left(x^{\prime}, t\right)=W\left(x^{\prime}, t, 0\right)=\int v\left(x^{\prime}-t y^{\prime}, 0\right) \phi\left(y^{\prime}\right) d y^{\prime}
$$

verifies

$$
|V|_{a}^{(-b)} \leq C|v|_{b}
$$

for $a \geq b \geq 0$. But, as already pointed out, when $b$ is an integer a function $v \in H_{b+\delta}^{(-b)}, \delta>0$ - the case which will concern us in the proof of Theorem 1may not belong to $H_{b}$, so that we cannot avail ourselves of the above inequality. Let us therefore modify the approach of [1], as follows.

Let $\phi$ be symmetric with respect to each coordinate hyperplane of $\mathbb{R}^{N-1}$. For $t>0$ we have

$$
W_{x_{j}}\left(x^{\prime}, t, x_{N}\right)=t^{-1} \int v\left(x^{\prime}-t y^{\prime}, x_{N}\right) \phi^{(j)}\left(y^{\prime}\right) d y^{\prime}
$$

with $\phi^{(j)}=\phi_{y_{j}}$ if $j<N$, whereas

$$
W_{t}\left(x^{\prime}, t, x_{N}\right)=t^{-1} \int v\left(x^{\prime}-t y^{\prime}, x_{N}\right) \phi^{(N)}\left(y^{\prime}\right) d y^{\prime}
$$

with

$$
\phi^{(N)}\left(y^{\prime}\right)=(1-N) \phi\left(y^{\prime}\right)-\sum_{j=1}^{N-1} y_{j} \phi_{y_{j}}\left(y^{\prime}\right)
$$

-again a function which is symmetric with respect to each coordinate hyperplane of $\mathbb{R}^{N-1}$. All integrals $\int \phi^{(1)}\left(y^{\prime}\right) d y^{\prime}, \ldots, \int \phi^{(N)}\left(y^{\prime}\right) d y^{\prime}$ equal 0 , of course. Let us generalize all of this to higher order derivatives. With the standard multi-index notation we have

$$
D^{\alpha} W\left(x^{\prime}, t, x_{N}\right)=t^{-|\alpha|} \int v\left(x^{\prime}-t y^{\prime}, x_{N}\right) \phi_{\alpha}\left(y^{\prime}\right) d y^{\prime},
$$

where all functions $\phi_{\alpha} \in C_{c}^{\infty}\left(\mathbb{R}^{N-1}\right)$ satisfy $\int \phi_{\alpha}\left(y^{\prime}\right) d y^{\prime}=0$ provided that $|\alpha|>0$ (otherwise $\phi_{\alpha}$ would simply be $\phi$ ). In the particular case when $\alpha$ is of the form $(0, \ldots, 0, h)$, that is, when $D^{\alpha}$ is the $h$-th pure derivative with respect to $t$, the function $\phi_{\alpha}$ is symmetric with respect to each coordinate hyperplane of $\mathbb{R}^{N-1}$ and therefore satisfies

$$
\int y_{j} \phi_{\alpha}\left(y^{\prime}\right) d y^{\prime}=0 \quad \forall j=1, \ldots, N-1 \text {. }
$$


Let $x_{N}>0$. Let the multi-index $\beta$ be defined by the identity $D^{\beta}=D^{\alpha} \partial / \partial t$. Suppose that $v$ is in some space $H_{1+\delta}^{(-1)}, \delta>0$; then, if $\alpha=(0, \ldots, 0, h)$, the function $D^{\alpha} V_{t}\left(x^{\prime}, t, x_{N}\right)$ equals $t^{-|\alpha|-1} \int v\left(x^{\prime}-t y^{\prime}, x_{N}\right) \phi_{\beta}\left(y^{\prime}\right) d y^{\prime}$ as well as $-t^{-|\alpha|} \int y^{\prime} \cdot \nabla v\left(x^{\prime}-t y^{\prime}, x_{N}\right) \phi_{\alpha}\left(y^{\prime}\right) d y^{\prime}$ (with $\nabla=$ gradient in the first $N-1$ variables). This leads to the identities

$$
\begin{gathered}
\int v\left(x^{\prime}-t y^{\prime}, x_{N}\right) \phi_{\beta}\left(y^{\prime}\right) d y^{\prime}=-t \int y^{\prime} \cdot \nabla v\left(x^{\prime}-t y^{\prime}, x_{N}\right) \phi_{\alpha}\left(y^{\prime}\right) d y^{\prime} \\
=-t \int y^{\prime} \cdot\left[\nabla v\left(x^{\prime}-t y^{\prime}, x_{N}\right)-\nabla v\left(x^{\prime}, x_{N}\right)\right] \phi_{\alpha}\left(y^{\prime}\right) d y^{\prime}
\end{gathered}
$$

where (11) has been utilized. If instead $\alpha_{j}>0$ for some $j<N$ we write $D^{\beta}$ as $D^{\alpha^{\prime}} \partial / \partial x_{j}$ and obtain

$$
\begin{gathered}
\int v\left(x^{\prime}-t y^{\prime}, x_{N}\right) \phi_{\beta}\left(y^{\prime}\right) d y^{\prime}=t \int v_{x_{j}}\left(x^{\prime}-t y^{\prime}, x_{N}\right) \phi_{\alpha^{\prime}}\left(y^{\prime}\right) d y^{\prime} \\
=t \int\left[v_{x_{j}}\left(x^{\prime}-t y^{\prime}, x_{N}\right)-v_{x_{j}}\left(x^{\prime}, x_{N}\right)\right] \phi_{\alpha^{\prime}}\left(y^{\prime}\right) d y^{\prime}
\end{gathered}
$$

since $\left|\alpha^{\prime}\right|>0$.

Lemma. For $b \in \mathbb{N}, 0<\delta<1$ let $v \in H_{b+\delta}^{(-b)}$ and have support in $\frac{1}{2} \bar{B}^{+}$. The function $V\left(x^{\prime}, x_{N}\right)=\int_{\mathbb{R}^{N-1}} v\left(x^{\prime}-x_{N} y^{\prime}, 0\right) \phi\left(y^{\prime}\right) d y^{\prime}$ is in any space $H_{a}^{(-b)}$ for $a \geq b+\delta$, and

$$
|V|_{a}^{(-b)} \leq C|v|_{b+\delta}^{(-b)}
$$

Moreover, $V\left(x^{\prime}, 0\right)=v\left(x^{\prime}, 0\right)$ if $\int \phi\left(y^{\prime}\right) d y^{\prime}=1$.

Proof. For $h+b+\delta \geq a$ we have $H_{a}^{(-b)} \supseteq H_{h+b+\delta}^{(-b)}$, so that it is not restrictive to suppose $a=h+b+\delta$.

Let $b=1$. Since $v(\cdot, 0)$ is of class $H_{1-\delta}$, it follows from Lemma 2.3 of [1] that

$$
|V|_{h+1}^{(-1+\delta)} \leq C|v|_{1-\delta} \leq C|v|_{1+\delta}^{(-1)}
$$

This yields a bound of the required type on $\sigma^{h+\delta}|V|_{h+1 ; B_{[\sigma]}^{+}}$for $h$ a nonnegative integer. (Notice that $V\left(x^{\prime}, x_{N}\right)$ is smooth for $x_{N}>0$, so that we need only worry about its behavior as $x_{N} \rightarrow 0$.) Let us proceed to bound

$$
\sigma^{h+\delta} \sup _{x, \xi \in B_{[\sigma]}^{+}, x \neq \xi}\left|D^{\alpha} V(\xi)-D^{\alpha} V(x)\right||\xi-x|^{-\delta}
$$

for $|\alpha|=h+1$. We take $|v|_{1+\delta}^{(-1)}=1$ and fix $x_{N}>0$.

First of all, we notice that

$$
\int_{0}^{x_{N}}\left|v_{s}\left(\xi^{\prime}-x_{N} y^{\prime}, s\right)-v_{s}\left(x^{\prime}-x_{N} y^{\prime}, s\right)\right| d s \leq C\left|\xi^{\prime}-x^{\prime}\right|^{\delta} x_{N}^{1-\delta} .
$$

On the other hand, since

$$
\int v\left(\xi^{\prime}, x_{N}\right) \phi_{\alpha}\left(y^{\prime}\right) d y^{\prime}=\int v\left(x^{\prime}, x_{N}\right) \phi_{\alpha}\left(y^{\prime}\right) d y^{\prime}=0
$$


we have

$$
\begin{aligned}
& \left|\int\left[v\left(\xi^{\prime}-x_{N} y^{\prime}, x_{N}\right)-v\left(x^{\prime}-x_{N} y^{\prime}, x_{N}\right)\right] \phi_{\alpha}\left(y^{\prime}\right) d y^{\prime}\right| \\
& \quad=\left|\int \phi_{\alpha}\left(y^{\prime}\right) d y^{\prime} \int_{0}^{x_{N}} y^{\prime} \cdot\left[\nabla v\left(\xi^{\prime}-s y^{\prime}, x_{N}\right)-\nabla v\left(x^{\prime}-s y^{\prime}, x_{N}\right)\right] d s\right| \\
& \quad \leq C \int_{0}^{x_{N}}\left|\nabla v\left(\xi^{\prime}-s y^{\prime}, x_{N}\right)-\nabla v\left(x^{\prime}-s y^{\prime}, x_{N}\right)\right| d s \leq C\left|\xi^{\prime}-x^{\prime}\right|^{\delta} x_{N}^{1-\delta}
\end{aligned}
$$

(where, once again, $\nabla$ is the gradient in the first $N-1$ variables). Summing up, the difference

$$
\begin{aligned}
D^{\alpha} V\left(\xi^{\prime}, x_{N}\right)-D^{\alpha} V\left(x^{\prime}, x_{N}\right) \\
=x_{N}^{-|\alpha|} \int\left[v\left(\xi^{\prime}-x_{N} y^{\prime}, 0\right)-v\left(x^{\prime}-x_{N} y^{\prime}, 0\right)\right] \phi_{\alpha}\left(y^{\prime}\right) d y^{\prime} \\
=x_{N}^{-|\alpha|} \int\left\{v\left(\xi^{\prime}-x_{N} y^{\prime}, x_{N}\right)-v\left(x^{\prime}-x_{N} y^{\prime}, x_{N}\right)\right. \\
\left.\quad-\int_{0}^{x_{N}}\left[v_{s}\left(\xi^{\prime}-x_{N} y^{\prime}, s\right)-v_{s}\left(x^{\prime}-x_{N} y^{\prime}, s\right)\right] d s\right\} \phi_{\alpha}\left(y^{\prime}\right) d y^{\prime}
\end{aligned}
$$

satisfies

$$
\left|D^{\alpha} V\left(\xi^{\prime}, x_{N}\right)-D^{\alpha} V\left(x^{\prime}, x_{N}\right)\right| \leq C\left|\xi^{\prime}-x^{\prime}\right|^{\delta} x_{N}^{1-|\alpha|-\delta} .
$$

Let us now pass to consider (for $\xi_{N}>x_{N}$ ) the difference

$$
\begin{gathered}
D^{\alpha} V\left(\xi^{\prime}, \xi_{N}\right)-D^{\alpha} V\left(\xi^{\prime}, x_{N}\right)=\int_{x_{N}}^{\xi_{N}} D^{\beta} V\left(\xi^{\prime}, t\right) d t \\
=\int_{x_{N}}^{\xi_{N}} t^{-|\alpha|-1} d t \int v\left(\xi^{\prime}-t y^{\prime}, 0\right) \phi_{\beta}\left(y^{\prime}\right) d y^{\prime}
\end{gathered}
$$

$D^{\beta}=D^{\alpha} \partial / \partial t$. Since

$$
\int v\left(\xi^{\prime}, x_{N}\right) \phi_{\beta}\left(y^{\prime}\right) d y^{\prime}=\int v\left(\xi^{\prime}, 0\right) \phi_{\beta}\left(y^{\prime}\right) d y^{\prime}=0,
$$

we have

$$
\begin{aligned}
\int v\left(\xi^{\prime}-t y^{\prime}, 0\right) \phi_{\beta}\left(y^{\prime}\right) d y^{\prime}= & \int\left\{v\left(\xi^{\prime}-t y^{\prime}, x_{N}\right)\right. \\
& \left.-\int_{0}^{x_{N}}\left[v_{s}\left(\xi^{\prime}-t y^{\prime}, s\right)-v_{s}\left(\xi^{\prime}, s\right)\right] d s\right\} \phi_{\beta}\left(y^{\prime}\right) d y^{\prime}
\end{aligned}
$$

Either by (12) or by (12)',

$$
\left|\int v\left(\xi^{\prime}-t y^{\prime}, x_{N}\right) \phi_{\beta}\left(y^{\prime}\right) d y^{\prime}\right| \leq C t^{1+\delta} x_{N}^{-\delta}
$$

On the other hand,

$$
\int_{0}^{x_{i}}\left|v_{s}\left(\xi^{\prime}-t y^{\prime}, s\right)-v_{s}\left(\xi^{\prime}, s\right)\right| d s \leq C t^{\delta} x_{N}^{1-\delta}\left|y^{\prime}\right|^{\delta}
$$


and therefore

$$
\left|\int \phi_{\beta} d y^{\prime} \int_{0}^{x_{N}}\left[v_{s}\left(\xi^{\prime}-t y^{\prime}, s\right)-v_{s}\left(\xi^{\prime}, s\right)\right] d s\right| \leq C t^{1+\delta} x_{N}^{-\delta}
$$

for $t \geq x_{N}$. Thus, (14)-(17) yield

$$
\begin{aligned}
\left|D^{\alpha} V\left(\xi^{\prime}, \xi_{N}\right)-D^{\alpha} V\left(\xi^{\prime}, x_{N}\right)\right| & \leq C\left(\int_{x_{N}}^{\xi_{N}} t^{-|\alpha|+\delta} d t\right) x_{N}^{-\delta} \\
& \leq C x_{N}^{-\delta}\left|\xi_{N}^{1-|\alpha|+\delta}-x_{N}^{1-|\alpha|+\delta}\right| .
\end{aligned}
$$

But for $x_{N} \leq \xi_{N} \leq 2 x_{N}$ we have

$$
\begin{aligned}
\left|\xi_{N}^{1-|\alpha|+\delta}-x_{N}^{1-|\alpha|+\delta}\right| x_{N}^{-\delta} & \leq C\left[x_{N}+\theta\left(\xi_{N}-x_{N}\right)\right]^{-|\alpha|+\delta}\left(\xi_{N}-x_{N}\right) x_{N}^{-\delta} \\
& \leq C x_{N}^{-|\alpha|}\left(\xi_{N}-x_{N}\right) \leq C x_{N}^{1-|\alpha|-\delta}\left(\xi_{N}-x_{N}\right)^{\delta}
\end{aligned}
$$

for some $\theta \in] 0,1$, whereas for $\xi_{N}>2 x_{N}$, which implies

$$
\xi_{N}^{\delta}+x_{N}^{\delta} \leq 2 \xi_{N}^{\delta} \leq 2^{1+\delta}\left(\xi_{N}-x_{N}\right)^{\delta},
$$

we have

$$
x_{N}^{-\delta}\left|\xi_{N}^{1-|\alpha|+\delta}+x_{N}^{1-|\alpha|+\delta}\right| \leq C x_{N}^{-\delta}\left(\xi_{N}^{\delta} x_{N}^{1-|\alpha|}+x_{N}^{\delta} x_{N}^{1-|\alpha|}\right) \leq C x_{N}^{1-|\alpha|-\delta}\left(\xi_{N}-x_{N}\right)^{\delta} .
$$

Thus,

$$
\left|D^{\alpha} V\left(\xi^{\prime}, \xi_{N}\right)-D^{\alpha} V\left(\xi, x_{N}\right)\right| \leq C x_{N}^{1-|\alpha|-\delta}\left(\xi_{N}-x_{N}\right)^{\delta}
$$

The required bound on

$$
x_{N}^{|\alpha|-1+\delta}\left|D^{\alpha} V\left(\xi^{\prime}, \xi_{N}\right)-\dot{D}^{\alpha} V\left(x^{\prime}, x_{N}\right)\right||\xi-x|^{-\delta}
$$

follows from (13) and (18). In the case $b>1$ it suffices to write

$$
V_{x_{i}}\left(x^{\prime}, x_{N}\right)=\int v_{x_{i}}\left(x^{\prime}-x_{N} y^{\prime}, 0\right) \phi\left(y^{\prime}\right) d y^{\prime}
$$

for $i<N$ and proceed by induction.

The last statement is obvious.

\section{Proofs of Theorems 1 AND 2}

If $1 \leq b \leq a<2$ the proof of (8) is achieved by repeating, with very minor changes, Step 1 (for $p=2$ ) and Step 2 of the proof of [6, Theorem 1], where the same norm estimate, for $A^{i j}=\delta^{i j}$, was proven in the homogeneous Dirichlet case-that is, with the solution $u$ and the test-functions $v$ all vanishing on $S^{0}$.

For a noninteger $a>2$, however, the above procedure no longer applies. And it is here that the lemma of the preceeding section comes into play. If $b$ remains in $[1,2[$, indeed, it enables us to utilize the previous step, which yields a bound

$$
|u|_{a^{\prime}}^{(-b)} \leq C \sum\left|f^{i}\right|_{a^{\prime}-1}^{(1-b)} \leq C \sum\left|f^{i}\right|_{a-1}^{(1-b)}
$$

for any noninteger $a^{\prime} \in[b, 2[$. Let, according to our lemma (for the case $b=1<a^{\prime}=1+\delta<2$; the case $1<b<2$ is [1, Lemma 2.3] for the choice of $\left.a^{\prime}=b\right) U \in H_{a}^{(-b)}$ equal $u$ on $S^{0}$, with supp $U \subseteq \frac{1}{2} \bar{B}^{+}$and

$$
|U|_{a}^{(-b)} \leq C|u|_{a^{\prime}}^{(-b)}
$$


The function $U^{\prime}=u-U$ vanishes on $S^{0}$ and satisfies

$$
\int_{B^{+}} a^{i j} U_{x_{i}}^{\prime} v_{x_{j}} d x=\int_{B^{+}}\left(f^{i}-a^{i j} U_{x_{j}}\right) v_{x_{i}} d x \quad \forall v \in C_{c}^{0}\left(B^{+}\right),
$$

the matrix $\left[a^{i j}\right]$ being the symmetric part of the matrix $\left[A^{i j}\right]$. This means that [6, Theorem 1] can be applied to $U^{\prime}$ (cf. (4)). Thus,

$$
\left|U^{\prime}\right|_{a}^{(-b)} \leq C \sum\left(\left|f^{i}\right|_{a-1}^{(1-b)}+\left|U_{x_{i}}\right|_{a-1}^{(1-b)}\right) .
$$

The result in the case at hand follows immediately once the inequalities

$$
|u|_{a}^{(-b)} \leq|U|_{a}^{(-b)}+\left|U^{\prime}\right|_{a}^{(-b)}, \quad\left|U_{x_{i}}\right|_{a-1}^{(1-b)} \leq|U|_{a}^{(-b)}
$$

and (19) have been taken into account. (See the proof of the implication $(5) \Rightarrow$ (4) in §2.) The proof of (8) for $b=k+b^{\prime}, 1 \leq b^{\prime}<2$, is easily obtained by induction on $k \in \mathbb{N}$, assuming the result true for $k-1$ and applying it to the derivatives $u_{x_{s}}, s=1, \ldots, N-1$, which satisfy the same identity as $u$ except for the $f^{i}$ 's replaced by the $f_{x_{s}}^{i}$ 's. Thus,

$$
\left|U_{x_{s}}\right|_{a-1}^{(1-b)} \leq C \sum\left|F_{x_{s}}^{i}\right|_{a-2}^{(2-b)}
$$

the above bound and the equation in $B^{+}$for what concerns $u_{x_{N} x_{N}}$ provide a bound of the required type on all derivatives of $u$ involved in the statement. Since the unweighted theory provides also a bound on, say, the $H_{0}$ norm of $u$, we have proved Theorem 1 .

Let us pass to the proof of Theorem 2. For simplicity's sake we assume that $a_{N N}=1$. Let

$$
A^{i j}=a^{i j}+\left(\delta^{i N}-\delta^{j N}\right) a^{i j} \quad\left(\text { so that } a^{N N}=1 \Rightarrow A^{i N}=\delta^{i N}\right):
$$

(i) is equivalent to

$$
\int_{B^{+}} A^{i j} u_{x_{i}} v_{x_{j}} d x=\int_{B^{+}}\left(f v+G_{x_{N}} v+G v_{x_{N}}\right) d x \quad \forall v \in C^{1}\left(\bar{B}^{+}\right),
$$

where

$$
\left.G \in H_{a-1}^{(1-b)} \quad \text { (so that } \operatorname{grad} G \in H_{a-2}^{(2-b)}\right) \text { with }\left.G\right|_{S^{0}}=g,|G|_{a-1}^{(1-b)} \leq C|g|_{b-1 ; S^{0}}
$$

(cf. [1, Lemma 2.3] again).

Let $U^{s}=u_{x_{s}}$. On one hand, as is done in [3], we utilize the Dirichlet problem satisfied by $U^{N}$; on the other hand, instead, we view $U^{1}, U^{2}, \ldots, U^{N-1}$ as solutions to oblique derivative problems. Here are the details.

$U^{N}$ satisfies

$$
\int_{B^{+}} a^{i j} U_{x_{i}}^{N} v_{x_{j}} d x=-\int_{B^{+}} f v_{x_{N}} d x \quad \forall v \in C_{c}^{1}\left(B^{+}\right)
$$

as well as $U^{N}(\cdot, 0)=g$ on $S^{0}$, so that the estimate for nonhomogeneous Dirichlet problems in variational form yields

$$
\left|U^{N}\right|_{a-1}^{(1-b)} \leq C\left(|f|_{a-2}^{(2-b)}+|g|_{b-1} ; S^{0}\right)
$$

(cf. (3)). For $s=1, \ldots, N-1, U^{s}$ satisfies

$$
\int_{B^{+}} A^{i j} U_{x_{i}}^{s} v_{x_{j}} d x=\int_{B^{+}}\left(-f v_{x_{s}}+G_{x_{s}} v_{x_{N}}-G_{x_{N}} v_{x_{s}}\right) d x \quad \forall v \in C^{1}\left(\bar{B}^{+}\right),
$$


so that Theorem 1 yields

$$
\left|U^{s}\right|_{a-1}^{(1-b)} \leq C\left(|f|_{a-2}^{(2-b)}+|\operatorname{grad} G|_{a-2}^{(2-b)}\right) \leq C\left(|f|_{a-2}^{(2-b)}+|g|_{b-1 ; S^{0}}\right) .
$$

Thanks to the bound on $|u|_{0}$ provided by the unweighted theory, (10) has been proven.

\section{REFERENCES}

1. D. Gilbarg and L. Hörmander, Intermediate Schauder estimates, Arch. Rational Mech. Anal. 74 (1980), 297-318.

2. G. M. Lieberman, Intermediate Schauder estimates for oblique derivative problems, Arch. Rational Mech. Anal. 93 (1986), 129-134.

3. $\ldots$, Oblique derivative problems in Lipschitz domains. I-Continuous boundary data, Boll. Un. Mat. Ital. B (7) 1 (1987), 1185-1210.

4. J. Pipher, Oblique derivative problems for the Laplacian in Lipschitz domains, Rev. Mat. Iberoamericana 3 (1987), 455-472.

5. G. M. Troianiello, Elliptic differential equations and obstacle problems, Plenum Press, New York, 1987.

6. _ Estimates of the Caccioppoli-Schauder type in weighted function spaces, Trans. Amer. Math. Soc. 334 (1992), 551-573.

Dipartimento di Matematica, Istituto "Guido Castelnuovo", Università degli Studi di Roma, "La Sapienza", Piazzale Aldo Moro 2, I - 00185 Roma, Italy 